\title{
Twin pregnancy complicated with congenital Hemivertebra: report of two cases and literature review
}

Tingting $X u^{1,2}$, Xiaodong Wang ${ }^{1,2}$, Haiyan $Y u^{1,2^{*}}$ (D) and Fumin Zhao ${ }^{1,2,3^{*}}$

\begin{abstract}
Background: Hemivertebra deformity, involving one or multiple vertebral bodies, is one of the important causes of congenital scoliosis. Congenital fetal hemivertebrae could be diagnosed by ultrasonography and confirmed by fetal magnetic resonance imaging during pregnancy. However, reports of hemivertebrae in twins during the perinatal period are very rare.

Case presentation: We report two cases of congenital fetal hemivertebrae, each affecting one fetus in a dichorionic diamniotic (DCDA) twin pregnancy. We have also conducted a literature review of its prenatal screening, diagnosis, management, and outcomes. These two cases of congenital fetal hemivertebrae in one fetus of a DCDA twin were both initially found by ultrasonography and confirmed by fetal magnetic resonance imaging (MRI). One couple chose selective termination of the hemivertebrae fetus after they were extensively counseled by the multidisciplinary team regarding the treatment and prognosis of the hemivertebrae twin, and a healthy baby weighing $2320 \mathrm{~g}$ was delivered at the $37^{+1}$ gestational week. The other couple decided to continue the twin pregnancy and gave birth to two living newborns weighing $2580 \mathrm{~g}$ and $2060 \mathrm{~g}$ at $37^{+1}$ gestational weeks. These three babies were all in good health during follow-up.
\end{abstract}

Conclusions: Based on our center's experience, comprehensive ultrasonography is necessary for early prenatal diagnosis of this condition. In addition, fetal MRI will confirm the diagnosis of hemivertebrae and provide parents with helpful information for their decision about the fate of the affected fetus.

Keywords: Fetal hemivertebrae, Dichorionic diamniotic twin pregnancy, Magnetic resonance imaging

\section{Background}

Hemivertebra is a rare congenital vertebral abnormality that can lead to deformation of the spine, such as scoliosis, lordosis, or kyphosis [1], and it is usually located in the thoracic and lumbar regions. The birth rate of hemivertebrae is $0.05-0.10 \%$ [2], occurring more commonly in females. The sex ratios (male/female) for multiple vertebral anomalies and solitary vertebral anomalies are respectively 0.31 and 0.68 [3].

*Correspondence: fanjy422@163.com; hxzfm@sina.com

'Department of Obstetrics and Gynecology, West China Second University Hospital, Sichuan University, Chengdu, China

Full list of author information is available at the end of the article
The majority of published papers about hemivertebrae involve surviving newborns after birth, and few papers have focused on cases of congenital fetal hemivertebrae diagnosed during prenatal care. Most fetal hemivertebrae cases involve a singleton pregnancy, and cases of only one twin with hemivertebrae are very rare.

Based on the published data of fetal hemivertebra in singletons we can see that fetal hemivertebra can be divided into isolated cases and coexisting anomalies. Most fetuses with prenatally diagnosed hemivertebrae in singletons usually have coexisting anomalies, which could affect the prognosis of hemivertebra with high rates of cesarean delivery, growth restriction, and fetal/neonatal

(c) The Author(s). 2020 Open Access This article is licensed under a Creative Commons Attribution 4.0 International License, which permits use, sharing, adaptation, distribution and reproduction in any medium or format, as long as you give appropriate credit to the original author(s) and the source, provide a link to the Creative Commons licence, and indicate if changes were made. The images or other third party material in this article are included in the article's Creative Commons licence, unless indicated otherwise in a credit line to the material. If material is not included in the article's Creative Commons licence and your intended use is not permitted by statutory regulation or exceeds the permitted use, you will need to obtain permission directly from the copyright holder. To view a copy of this licence, visit http://creativecommons.org/licenses/by/4.0/ The Creative Commons Public Domain Dedication waiver (http://creativecommons.org/publicdomain/zero/1.0/) applies to the data made available in this article, unless otherwise stated in a credit line to the data. 
loss. Besides, nonisolated hemivertebrae of neonates usually born before term with higher mortality rates [4, 5]. The prognosis of fetal hemivertebra is related to the type, site, the number of the affected vertebra, and the associated anomalies. Isolated fetal hemivertebrae is considered to carry a good prognosis [6]. The presence of associated anomalies reduces the survival to $50 \%$, and when accompanied by significant oligohydramnios, the mortality is $100 \%$ [6].

Here, we reported two cases of one fetus with congenital hemivertebrae in dichorionic diamniotic (DCDA) twin pregnancies at West China Second University Hospital, a tertiary referral center in west China, between January 2018 to March 2020. Additionally, we conducted a literature review about the perinatal management and postnatal outcomes of twin pregnancies compared with one fetus with prenatally diagnosed hemivertebrae.

\section{Case presentation}

Case 1

A 32-year-old woman, gravida 2, para 0, conceived dichorionic diamniotic (DCDA) twins by ovulation-induction therapy. The couple was not consanguineous and had no reported history of medication, substance abuse, or a family history of congenital anomalies. The patient's serology was negative for HIV, VDRL, and HBsAg.

Prenatal ultrasonography identified the twin pregnancy with one fetus displaying hemivertebrae at $25^{+}$weeks of gestation. Given this condition, the patient was transferred to our department. The ultrasonography in our hospital revealed the hemivertebrae in the lumbar (L) 23 vertebral bodies in one twin (Fig. 1 panel 1), while the other twin was healthy. Prenatal diagnosis of the fetus with hemivertebrae was subsequently confirmed by fetal magnetic resonance imaging (MRI), which was shown in Additional file 1, besides, the fetal MRI of these twin was shown in Additional file 2. The spine in the thoracolumbar junction area was slightly affected by scoliosis and the vertebral body morphological structures of L1 and L2 were abnormal with no other structural abnormalities, including musculoskeletal, genitourinary, cardiac, and so on.

The chromosomal microarray analysis of the amniotic fluid sample from both twins was normal. In addition, amniotic fluid samples from the two fetuses identified them as nonidentical twins. After the couple was extensively counseled by a multidisciplinary team regarding the treatment and prognosis of the hemivertebrae twin, the parents chose selective termination of the hemivertebrae fetus. Thus, legal termination of the affected fetus was performed by ultrasound-guided intrathoracic injection of $\mathrm{KCl}$ during the pregnancy based on local laws and religious beliefs.

At $37^{+1}$ gestational week, a cesarean section was performed due to a breech presentation in labor. A healthy female baby weighing $2320 \mathrm{~g}$ was delivered with Apgar scores of 10 and 10 at 1 and $5 \mathrm{~min}$, respectively. The dead co-twin was a male baby weighing $1655 \mathrm{~g}$. The parents agreed to postmortem radiological examination but refused an autopsy of the dead co-twin. The normal twin was healthy with no abnormality. The radiological examination confirmed the dead co-twin had hemivertebrae (Fig. 1 panel 2), while the healthy baby had a normal spine. The baby was in good health for more than 2 years of follow-up.

\section{Case 2}

A 32-year-old pregnant woman, gravida 3, para 0, underwent in vitro fertilization and embryo transfer (IVF-ET), and 2 embryos were transferred to the uterus. After the embryo transfer, ultrasonography revealed a DCDA twin pregnancy. The patient's serology was negative for HIV, VDRL, and HBsAg. The couple had no reported history of medication, substance abuse, or a family history of

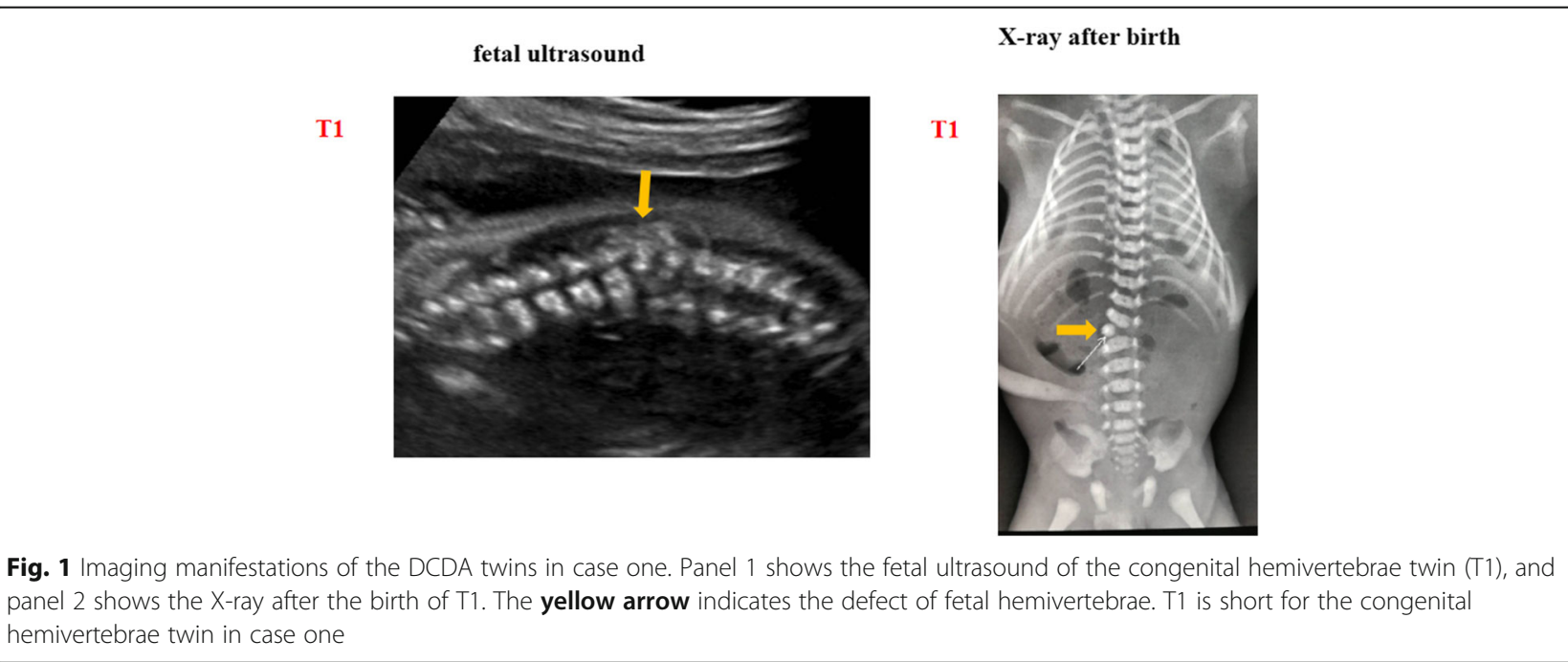


congenital anomalies. The regular prenatal ultrasonography at $25^{+3}$ gestational weeks identified that the T12 vertebral body of the spine was slightly abnormal in one twin (Fig. 2 panel 1) without any other structural abnormalities including musculoskeletal, genitourinary, cardiac, etc., while the co-twin was normal. Fetal MRI of twin was performed (Additional file 3) and the diagnosis of fetal hemivertebrae was made for this affected fetus (Fig. 2 panel 2). The co-twin was normal. The chromosomal microarray analysis of the amniotic fluid sample in both twins was normal. The couple decided to continue the pregnancy after extensive counseling by the multidisciplinary team.

A cesarean section was conducted at $37^{+1}$ gestational weeks for a fetal breech presentation, and two living male babies were delivered. The birth weights of these two newborns were $2580 \mathrm{~g}$ and $2060 \mathrm{~g}$ with Apgar scores of 10 and 10 at 1 and $5 \mathrm{~min}$, respectively. We explained to the parents the advantages and disadvantages of ultrasound (US), Xray, and MRI examinations, and the parents were worried about the radiation of X-rays and the expense of MRI. Finally, they chose postnatal documentation of the hemivertebrae in the second twin by the postnatal US. We found the T12 level hemivertebrae (Fig. 2 panel 3) and no other structural abnormalities. The twins were in good health for more than 1 year of follow-up. The baby with hemivertebrae was assessed for associated skeletal, cardiac, renal, and gastrointestinal anomalies after birth and showed no progression of scoliosis. He is being followed by the Child Healthcare Department and Orthopedics Department.

\section{Discussion and conclusions}

Hemivertebrae may be isolated or found in association with coexisting multiple anomalies $[2,7]$. The exact etiology of hemivertebrae is unclear $[7,8]$, involving both genetic and environmental factors [9]. The incidence of karyotypic abnormalities in fetuses with isolated hemivertebrae is low $[2,8,10]$. In our study, chromosomal microarray analysis of the amniotic fluid sample from both twins of the included cases was conducted and no abnormal results were found.

Hemivertebrae is also a component of several genetic syndromes, including Jarcho-Levin syndrome (autosomal recessive; fused vertebrae, scoliosis, abnormal rib alignment), Klippel-Fiel syndrome (autosomal recessive or dominant; the fusion of the cervical vertebrae), VACT ERLsyndrome (sporadic; vertebral and ventricular septal defects, anal atresia, tracheoesophageal fistula, renal anomalies, radial dysplasia, and single umbilical artery), and the OEIS complex (sporadic; omphalocele, exstrophy of the cloaca, imperforate anus, and spinal defects) $[1,3,4,7,10,11]$. Therefore, it is very important to be specific about its identification and its correlations with other complications. Attention should be given to the clinical diagnosis and treatment of hemivertebrae. What's more, the high risk of associated anomalies needs to be correctly disclosed and reported to the parents prenatally at the diagnosis of hemivertebra.

Routine prenatal ultrasonographic screening is the primary method used to identify fetal hemivertebrae; however, the diagnosis of hemivertebrae should be confirmed by fetal MRI and then post-birth or post mortem by X-ray examinations. Additional structural abnormalities, mainly musculoskeletal, genitourinary, and cardiac, are found in more than $70 \%$ of cases $[2,5,7$, 12]. Therefore, assessments for associated skeletal, cardiac, renal, and gastrointestinal anomalies should be performed once hemivertebrae is recognized [13].

In addition, fetal MRI is very useful in evaluating the entire spine. It can detect some abnormalities, especially

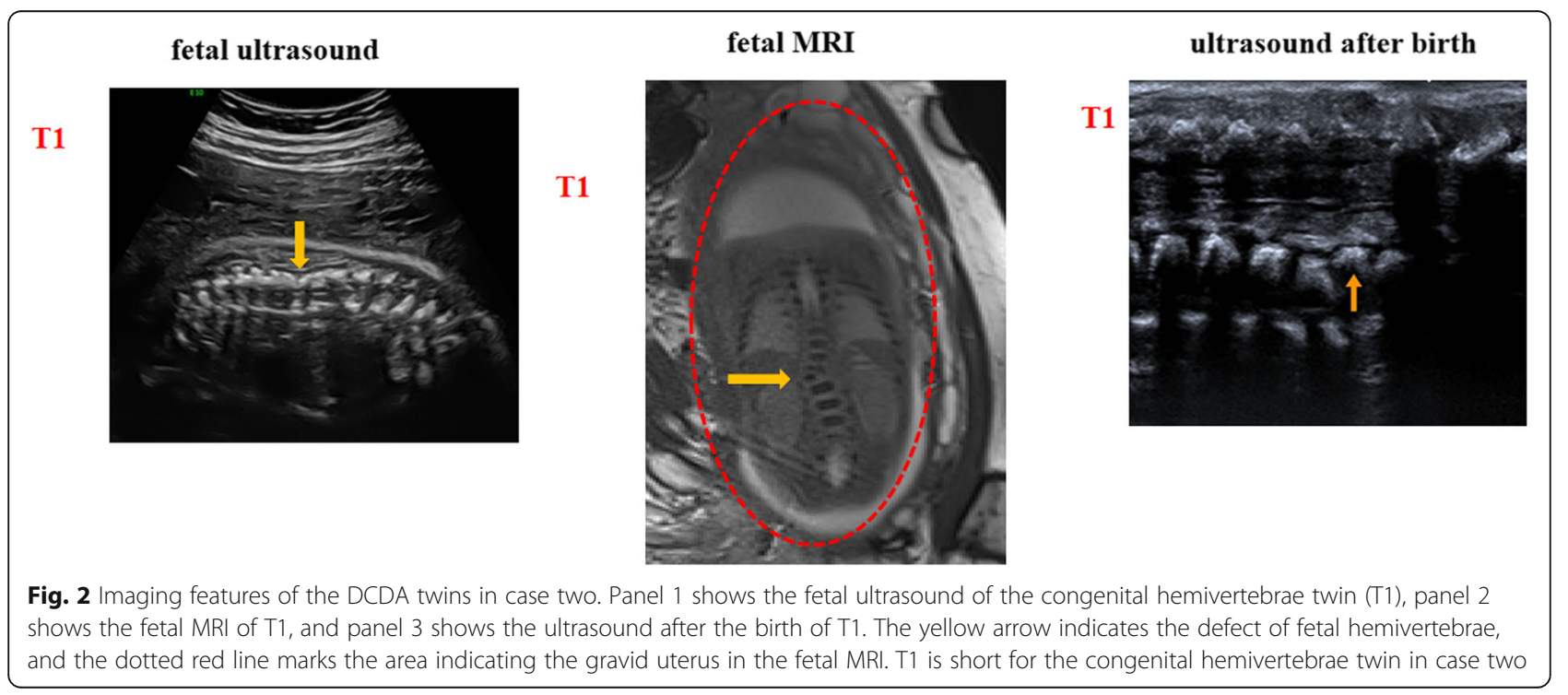




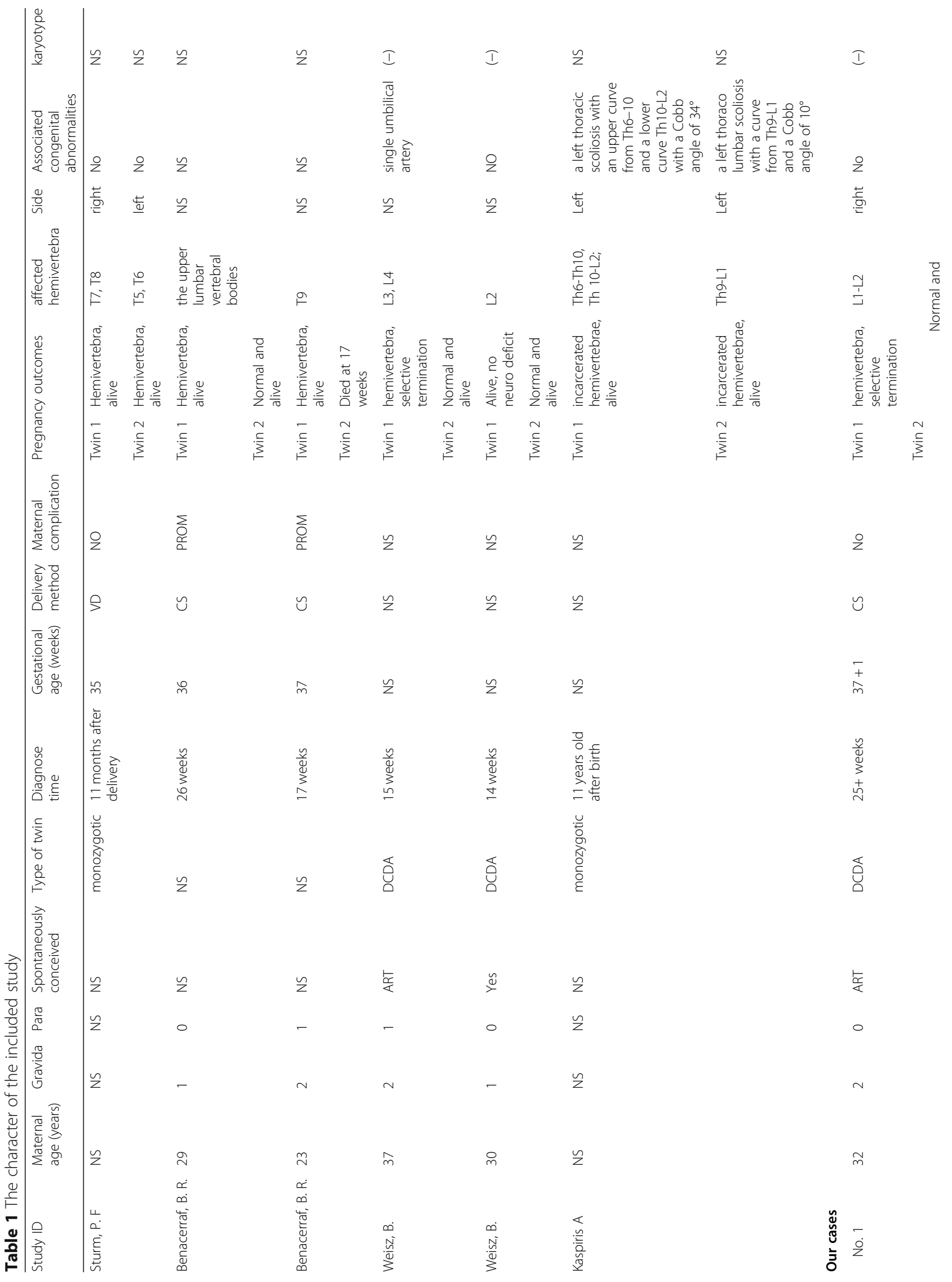




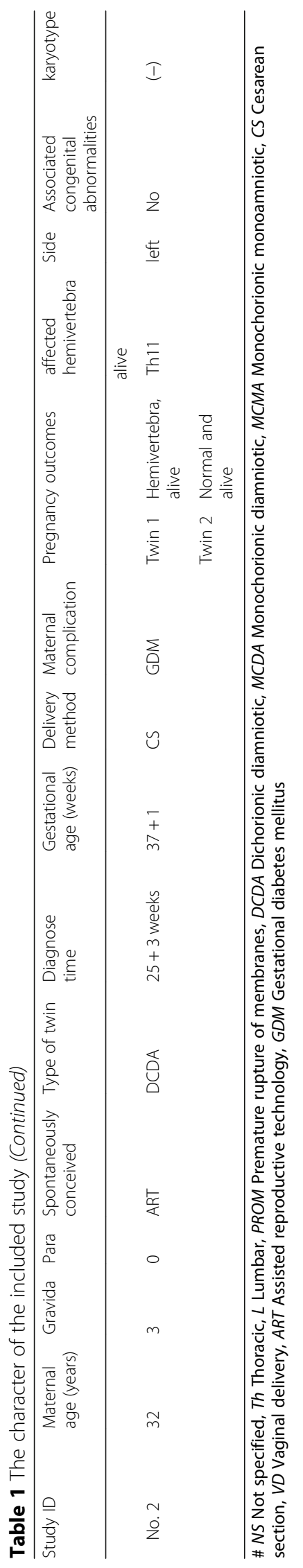


central nervous system abnormalities, and add some additional useful information in complicated cases, for which the information is insufficient by ultrasonography $[4,11]$. The fetal MRI results allow the fetal condition and prognosis to be evaluated in detail by a multidisciplinary team and further suggestions and advice can be given to the couple. In our study, fetal MRI of these two included cases was conducted to confirm the diagnosis of fetal hemivertebrae.

To the best of our knowledge, the globally published papers in English related to twin pregnancy with the hemivertebrae number only 4 so far $[9,14-16]$. It has been found that in twin pregnancy, even in identical twins, if one fetus has congenital scoliosis, the other one can be normal [9]. However, there is no consensus on the perinatal management of twin pregnancies with hemivertebrae.

Sturm P. F., et al. [9] reported a case of monozygotic twins who both suffered from hemivertebrae with no other deformity. These twins were first evaluated for their spinal anomalies at age 11 months; however, both twins showed minimal progression of their spinal curves within two years of follow-up. Benacerraf B. R., et al. [14] reported two cases of twin pregnancies affected by hemivertebrae. One case delivered one hemivertebrae newborn and one healthy newborn. However, the other case was a blighted twin and only a single fetus with hemivertebrae was alive. Weisz B, et al. [5] reported two cases of DCDA twin pregnancies with one hemivertebrae fetus and one healthy fetus. Among these two cases, one case chose selective termination, and the other case delivered two living newborns without any neurological deficits within a 24 month follow up. Kaspiris A, et al. [16] reported a case where both twins had hemivertebrae, with congenital scoliosis, moderate mental retardation, and dyslalia. Detailed information of these cases is shown in Table 1.

From the above-published results and our center's experience, we can see that all of these hemivertebrae cases were alive after birth except when the parents chose selective termination, and some had deformation of the spine; however, isolated hemivertebrae has a good prognosis. Hence, given the above available evidence, there is no different behavior of hemivertebra in twins as compared to singletons.

If only one vertebra is affected and no other fetal malformations are detected, conservative management of the pregnancy could be offered. Termination of pregnancy should only be offered if multiple vertebrae are malformed, or if anomalies in additional organ systems are seen [13]. The treatment and prognosis of hemivertebrae depend on the timing of its diagnosis, the location and number of the affected vertebra, and the associated anomalies $[2,8]$.
Fetuses with hemivertebrae have high rates of cesarean section, growth restriction, low birth weight, and/or preterm delivery [2]. In our study, these two cases were both delivered by cesarean section due to breech presentation of a twin pregnancy.

In conclusion, comprehensive ultrasonographic screening of the fetus allows an early prenatal diagnosis of hemivertebrae. The prognosis of isolated hemivertebrae is good and expectant management of the pregnancy can be adopted if no other major fetal malformations are detected. The prenatal ultrasound and MRI diagnosis of fetal hemivertebrae require careful and meticulous examinations, and this can provide parents with helpful information about their decision regarding the fate of the pregnancy. When the parents opt to continue with the pregnancy, a multidisciplinary team involving geneticists, obstetricians, pediatricians, neurosurgeons, spinal surgeons, physiotherapists, and psychologists is necessary. In addition, chromosomal analysis can be offered. Once identified, a careful neonatal assessment for associated cardiac and genitourinary anomalies needs to be performed. If there are no other complicating factors, standard management of labor and delivery is recommended.

\section{Supplementary information}

Supplementary information accompanies this paper at https://doi.org/10. 1186/s12884-020-03177-3.

Additional file 1. Fetal MRI of afftected fetus in case one.

Additional file 2. Fetal MRI of twin in case one.

Additional file 3. Fetal MRI of twin in case two.

\section{Abbreviations}

DCDA: Dichorionic diamniotic; MRI: Magnetic resonance imaging; HIV: Human immunodeficiency virus; VDRL: Venereal disease research laboratory; HBsAg: Hepatitis B surface antigen; IVF-ET: In vitro fertilization and embryo transfer

\section{Acknowledgments}

We feel grateful for the doctors and staff who have been involved in this work. All persons that contributed to this study are listed authors and meet the criteria for authorship.

\section{Authors' contributions}

XTT: Data collection and manuscript writing. WXD: Participated in the design of the study. ZFM: Participated in the manuscript revision. YHY: Project development, manuscript revision, and supervised the work. The authors have read and approved the manuscript.

\section{Funding}

This study was supported by the Academic and Technical Leader's Foundation of Sichuan Province (No.2017-919-25) and the Science Foundation of Sichuan Province (2018FZ0041). With the support of the first two funds (No.2017-919-25 and 2018FZ0041), we were able to design the study, complete the data collection and analysis.

Availability of data and materials Not applicable for this case series. 


\section{Ethics approval and consent to participate}

This study was approved by the ethical committees at the West China Second University Hospital of Sichuan University.

\section{Consent for publication}

Written informed consent was obtained from each couple for publication of potentially identifying images and clinical details of the patient and fetal individual identifiable data and images. A copy of the written consent is available for review by the Editor of this journal.

\section{Competing interests}

None of the authors has any conflict of interest to declare.

\section{Author details}

'Department of Obstetrics and Gynecology, West China Second University Hospital, Sichuan University, Chengdu, China. ${ }^{2}$ Key Laboratory of Birth Defects and Related Diseases of Women and Children (Sichuan University), Ministry of Education, Chengdu, China. ${ }^{3}$ Department of Radiology, West China Second University Hospital, Sichuan University, Chengdu, China.

Received: 21 May 2020 Accepted: 14 August 2020

Published online: 20 August 2020

\section{References}

1. Leung YL, Buxton N. Combined diastematomyelia and hemivertebra: a review of the management at a single Centre. J Bone Joint Surg Br. 2005; 87(10):1380-4

2. Varras M, Akrivis C. Prenatal diagnosis of fetal hemivertebra at 20 weeks' gestation with literature review. Int J Gen Med. 2010;3:197-201.

3. Wynne-Davies R. Congenital vertebral anomalies: aetiology and relationship to spina bifida cystica. J Med Genet. 1975;12(3):280-8.

4. Basude $S$, McDermott $L$, Newell $S$, et al. Fetal hemivertebra: associations and perinatal outcome. Ultrasound Obstet Gynecol. 2015;45(4):434-8.

5. Wax JR, Watson WJ, Miller RC, et al. Prenatal sonographic diagnosis of hemivertebrae: associations and outcomes. J Ultrasound Med. 2008;27(7): 1023-7.

6. Zelop CM, Pretorius DH, Benacerraf BR. Fetal hemivertebrae: associated anomalies, significance, and outcome. Obstet Gynecol. 1993;81(3):412-6.

7. Li Y, Choy KW, Xie HN, et al. Congenital hydrocephalus and hemivertebrae associated with de novo partial monosomy 6q (6q25.3 $\rightarrow$ qter). Balkan J Med Genet. 2015;18(1):77-84.

8. Goldstein I, Makhoul IR, Weissman A, Drugan A. Hemivertebra: prenatal diagnosis, incidence and characteristics. Fetal Diagn Ther. 2005;20(2):121-6.

9. Sturm PF, Chung R, Bomze SR. Hemivertebra in monozygotic twins. Spine (Phila Pa 1976). 2001;26(12):1389-91.

10. Bollini G, Docquier PL, Viehweger E, Launay F, Jouve JL. Thoracolumbar hemivertebrae resection by double approach in a single procedure: longterm follow-up. Spine (Phila Pa 1976). 2006:31(15):1745-57.

11. Chen M, Chan B, Lam TP, Shek T, Lee CP, Tang MH. Sonographic features of hemivertebra at 13 weeks' gestation. J Obstet Gynaecol Res. 2007;33(1):747

12. Alvarez de la Rosa M, Padilla Pérez Al, de la Torre Fernández de Vega FJ, et al. Genetic counseling in a case of congenital hemivertebrae. Arch Gynecol Obstet. 2009;280(4):653-8.

13. Johal J, Loukas M, Fisahn C, Chapman JR, Oskouian RJ, Tubbs RS. Hemivertebrae: a comprehensive review of embryology, imaging, classification, and management. Childs NervSyst. 2016:32(11):2105-9.

14. Benacerraf BR, Greene MF, Barss VA. Prenatal sonographic diagnosis of congenital hemivertebra. J Ultrasound Med. 1986;5(5):257-9.

15. Weisz B, Achiron R, Schindler A, Eisenberg VH, Lipitz S, Zalel Y. Prenatal sonographic diagnosis of hemivertebra. J Ultrasound Med. 2004:23(6):853-7.

16. Kaspiris A, Grivas TB, Weiss HR. Congenital scoliosis in monozygotic twins: case report and review of possible factors contributing to its development. Scoliosis. 2008;3:17.

\section{Publisher's Note}

Springer Nature remains neutral with regard to jurisdictional claims in published maps and institutional affiliations.

Ready to submit your research? Choose BMC and benefit from:

- fast, convenient online submission

- thorough peer review by experienced researchers in your field

- rapid publication on acceptance

- support for research data, including large and complex data types

- gold Open Access which fosters wider collaboration and increased citations

- maximum visibility for your research: over $100 \mathrm{M}$ website views per year

At $\mathrm{BMC}$, research is always in progress.

Learn more biomedcentral.com/submissions 